\title{
Transfer of Tetracycline-resistance between Strains of Staphylococcus aureus in Mixed Cultures
}

\author{
By R. W. LACEY \\ Department of Bacteriology, University of Bristol, Bristol, BS8 ITD
}

(Accepted for publication 26 August 197I)

\begin{abstract}
SUMMARY
A plasmid conferring tetracycline-resistance from a clinical isolate of Staphylococcus aureus (540I) was transferred to a wide variety of other strains by mixed culture in nutrient broth. Strain 609 converted to tet-r by this method could transfer tet-r efficiently (up to $10^{-3}$ in $12 \mathrm{~h}$.) to other strains. Co-factor studies on this donor suggested that transfer of tet-r is mediated by phage transduction. However, filtrates of the donor culture contained few particles able to transfer tetracycline-resistance. It is probable that the transfer occurs by short-lived or cell-bound phage particles. Filtrates of the donor strain obtained after induction with mitomycin $\mathrm{C}$ were able to transduce tetracycline-resistance, but at low frequency.

The gene(s) for tetracycline-resistance in these strains is probably borne by a plasmid, since they segregate tetracycline-sensitive derivatives. Ultraviolet (u.v.) light-inactivation studies on transduction of tet-r support this conclusion.
\end{abstract}

\section{INTRODUCTION}

In most studies of transduction in Staphylococcus aureus, genetic markers have been transferred to recipient strains from cell-free lysates containing a high-titre of bacteriophage particles. The relevance of this type of experiment to possible transfer of antibiotic-resistance under natural conditions is uncertain, but the transfer of resistance to erythromycin and penicillin (Novick \& Morse, 1967) and to neomycin (Lacey, I97I) occurs between mixed cells of staphylococci both in broth culture and in vivo under laboratory conditions. The transfer is probably mediated by transduction.

During the investigation of the transfer of neomycin-resistance between staphylococci (Lacey, I97I) it was noticed that tetracycline-resistance was transferred from one of the recipient strains to the neomycin-resistant donor strain in mixed cultures. This paper describes the investigation of this phenomenon. The frequency of transfer can be as high as one recipient in 800 acquiring tetracycline-resistance, and the mechanism of gene transfer is almost certainly transduction. Tetracycline-resistance was transferred successively to three strains; in each strain the genetic determinant is probably carried by a stable plasmid. These results provide additional evidence for the hypothesis that some antibiotic-resistance in naturally-occurring strains of Staphylococcus aureus has been acquired by transfer of extra-chromosomal elements.

\section{METHODS}

Strains of Staphylococcus aureus. The gene(s) for tetracycline-resistance in all the strains described here was transferred either directly or indirectly to them from strain 540I. This strain was isolated in 1954 from the wound of an in-patient in Bristol, immediately freeze- 
dried, and kindly provided by Dr V. G. Alder. Viable bacteria were recovered from a freeze-dried ampoule in June 1969 and a single-colony isolate maintained on an agar slope at room temperature. The minimum inhibitory concentration (MIC) of tetracycline was $100 \mu \mathrm{g} . / \mathrm{ml}$. Tetracycline-resistance was transferred from strain 540I in mixed broth cultures successively to strains 609, 6936, and then to 1030. Strain 609 is identical to strain $609 \mathrm{~N}_{\mathrm{D}}$ (Lacey, 197I). Strain 6936 is an isolate from the normal skin of an in-patient, and strain 1030 is a laboratory culture originally obtained from Colindale and is not detectably lysogenic (Novick, 1967).

Other recipient strains were the propagating strains (PS) of phages 47,54 and $83 \mathrm{~A}$ in the International Basic Set, and 5 recently isolated cultures from clinical sources. From these, one-step mutants resistant to streptomycin (100 $\mu \mathrm{g} . / \mathrm{ml}$.) or novobiocin $(5 \mu \mathrm{g} . / \mathrm{ml}$.) were obtained by plating the strains on nutrient agar containing the appropriate antibiotics.

Media. Nutrient broth was double strength Bacto (Difco) with the addition of $0.5 \%(\mathrm{w} / \mathrm{v})$ $\mathrm{NaCl}$. Nutrient agar was prepared from Oxoid blood agar no. 2.

Transfer of tetracycline-resistance in mixed cultures. Overnight broth cultures (I ml.) of the tet-r donor strain and the recipient strain resistant to either streptomycin or novobiocin were added to $100 \mathrm{ml}$. nutrient broth $+0.01 \mathrm{M}$-calcium chloride (calcium broth) in $250 \mathrm{ml}$. conical flasks and shaken at $35^{\circ}$. After $20 \mathrm{~h}$. samples of the culture were plated on nutrient agar containing Io $\mu \mathrm{g}$. tetracycline and either $100 \mu \mathrm{g}$. streptomycin or $5 \mu \mathrm{g}$. novobiocin $/ \mathrm{ml}$. to select tetracycline-resistant recipient cells. As controls in each experiment, the donor and recipient strains were each incubated singly and plated onto the selective media. No mutant recipient strains resistant to Io $\mu \mathrm{g}$. tetracycline/ml. were isolated throughout the experiments. Mutant donor strains resistant to either $100 \mu \mathrm{g}$. streptomycin or to $5 \mu \mathrm{g}$. novobiocin/ $\mathrm{ml}$. were infrequent. Some of the colonies from each experiment were checked for colonial pigment on nutrient agar containing $1 \%$ glycerol monoacetate (Willis \& Turner, 1963), the Tween 80 reaction (Gonzales \& Sierra, 1961) haemolysin production, sensitivity to antibiotics, and bacteriophage type. The frequency of transfer of tetracycline-resistance was expressed as the number of tetracycline-resistant recipients in the total number of recipient colonies.

Bacteriophage typing was done by the method of Blair \& Williams (I96I) with the addition of three new typing phages, 83A, 84 and 85 (Report, 1967).

Antibiotic sensitivity was determined by Oxoid Multodiscs and the MIC of tetracycline for a proportion of colonies established by an agar plate method. Tetracycline was the hydrochloride, B.P., obtained from Berk Pharmaceuticals Ltd, Godalming, Surrey.

Mitomycin C induction. Details of this method were kindly provided by Dr D. J. Stickler. An overnight broth culture was diluted $\mathrm{I}$ in 20 into warmed nutrient broth and aerated at $35^{\circ}$ until early logarithmic phase (about 3 h.). Mitomycin C (Sigma Chemical Co., London S.W.6) was added to a final concentration of between $0 \cdot 1 \mu \mathrm{g}$. and $5 \mu \mathrm{g} . / \mathrm{ml}$. depending on the strain. Treated cultures were aerated at $35^{\circ}$ for $10 \mathrm{~min}$., centrifuged and the cells resuspended in fresh warmed nutrient broth. After about $2 \mathrm{~h}$. (at $35^{\circ}$ ) the culture cleared. The lysate was filtered through $0.45 \mu$ diam. 'millipore' filters and stored at $4^{\circ}$. The filtrate contained about $\mathrm{Io}^{9}$ plaque-forming units (p.f.u.) of phage $/ \mathrm{ml}$.

Transduction experiments employing mitomycin-induced lysates were carried out by the method of Dyke, Parker \& Richmond (1970). Recipient strains used were 609 and 6936. U.v. survival of lysates was measured using a 240 volt 'Hanovia' lamp at $25 \mathrm{~cm}$. for various times.

Lysogenation of strains with phage P609. Strain 609 contains a prophage (designated P609) of seriological group B (Lacey, 197I) which forms large plaques on PS 83A and converts 
the Tween 80 reaction from positive to negative on lysogenization of appropriate strains. In these features this phage resembles the $\beta$ phages in staphylococci epidemic in Danish hospitals (Bülow, 1970).

Phage P609 was isolated from a mitomycin C-induced lysate of strain 609 which was diluted and spotted onto the surface of an agar plate seeded with PS 83A. After incubation, a single plaque was picked into broth and again spotted onto PS 83A. The resultant plaque morphology was uniform. The phage was then propagated further on Ps $83 \mathrm{~A}$, harvested by the freeze-thawing method of Williams \& Rippon (1952) and stored at $4^{\circ}$.

Broth cultures of strains were lysogenized with phage P609 (multiplicity $\simeq 0 \cdot 3$ ). Proof of lysogenization was indicated by immunity to lysis by P609 and production of phage able to plate on the parent strain.

Removal of phage $P 609$ from strain 609 tet-r. Plates containing $40 \%$ milk agar,were seeded with an overnight broth culture of strain 609 and exposed to u.v. light for $20 \mathrm{sec}$. at $25 \mathrm{~cm}$. from a 240 volt 'Hanovia' lamp. After incubation for $48 \mathrm{~h}$. the resultant colonies were examined. The occasional Tween 80 positive mutant colonies of strain 609 were'smaller than the wild (Tween 80 negative) type (Lacey, Alder \& Gillespie, 1970). One of these was now lysed by phage P609 and not induced by mitomycin C. This strain is denoted as 609 tet-r, (P609) $)^{-}$. Lysogenization of 609 tet-r, (P609) ${ }^{-}$with $\mathrm{P} 609$ produced a culture indistinguishable from strain 609 tet-r.

Phage adsorption was demonstrated by adding phage P609 to overnight cultures of the organism at a multiplicity of about 0.3 . After the addition of $0.01 \mathrm{M}$-calcium, the mixtures were allowed to stand at $37^{\circ}$ for $15 \mathrm{~min}$., centrifuged at $3000 \mathrm{~g}$ for $5 \mathrm{~min}$. andiphage particles in the supernatant assayed. Cultures that adsorbed the phage reduced the free phage titre by about $80 \%$.

\section{RESULTS}

Transfer of tetracycline-resistance from strain 540I successively to strains 609, 6036 and I030. The determinant of a tetracycline-resistance was transferred successively in mixed cultures from strain 540 I to strains 609, 6936 and I030 (Table I). The tetracycline-resistant derivatives recovered in each step had retained their colonial pigment, Tween 80 reaction, haemolytic properties, sensitivity to antibiotics other than tetracycline and the bacteriophage type.

Nature of transfer vector. Since the highest frequency of transfer was from strain 609 tet-r to $6936 s t r-r$, a mixed culture of these strains was used to investigate the nature of the transfer vector. After about $\mathrm{I} h$. transfer of resistance began and was essentially complete by $\mathrm{I} 2 \mathrm{~h}$. (Fig. I). The highest proportion of the recipient 6936 acquiring tetracyclineresistance was $\mathrm{I}$ in 800 colony-forming units. The addition of $0.03 \mathrm{M}$-sodium citrate after $4 \mathrm{~h}$. incubation prevented further transfer, but the total number of donor and recipient cocci was unaltered at $48 \mathrm{~h}$. No transfer at all occurred if citrate was added simultaneously with the mixing of the cultures. In the absence of added calcium the rate of transfer fell to about $\mathrm{I} \times 1 \mathrm{I}^{-8}$. For the optimum rate of transfer, both the donor and recipient cultures had to be mixed when in an exponential growth phase.

Since most staphylococcal phages require calcium ions for adsorption (Rountree, 195I), tetracycline-resistance is probably transferred by a phage vector. This conclusion is supported by the finding that 609 tet-r, (P609) ${ }^{-}$cannot transfer tetracycline-resistance to 6936 str-r $\left(<\mathrm{I} \times \mathrm{IO}^{-12}\right)$. A P609 lysogen of 609 tet-r (P609) $)^{-}$on the other hand was able to transfer tetracycline-resistance to $6936 \mathrm{str}-\mathrm{r}$ at high frequency $\left(2 \times 10^{-5}\right)$.

Although transfer is dependent on the presence of P609 in the donor, it is uncertain 


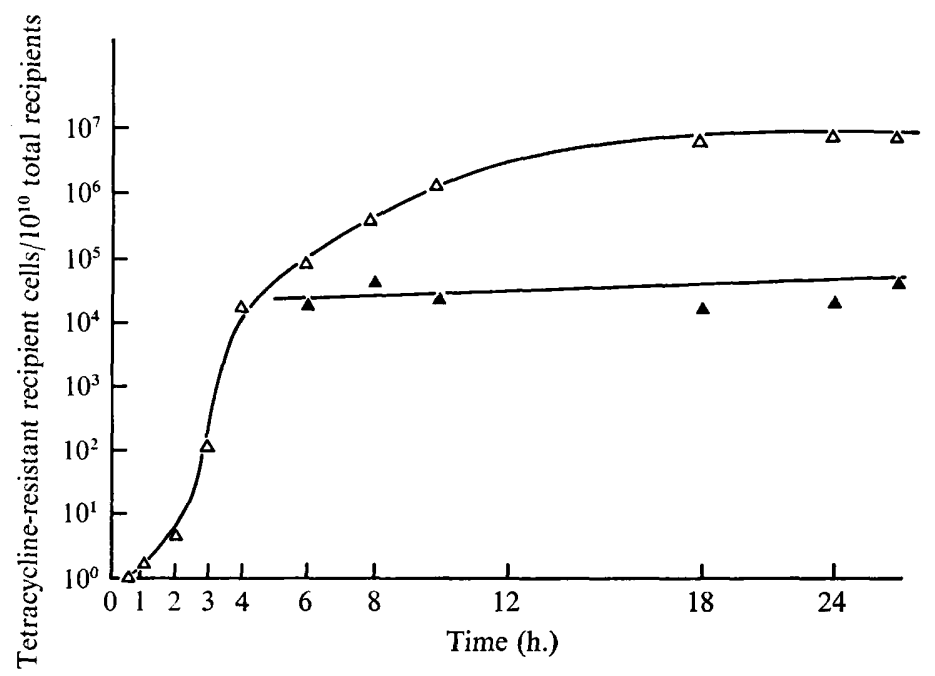

Fig. I. Transfer of tetracycline-resistance from donor culture 609 tet-r to recipient 6936 in $0.01 \mathrm{M}-$ calcium broth. $\triangle-\triangle$, Tetracycline-resistant recipients in broth without addition of citrate; $\Delta-\Lambda$, tetracycline-resistant recipients after addition of $0.03 \mathrm{M}$-citrate at $4 \mathrm{~h}$.

Table I. Properties of strains to which tetracycline-resistance was successively transferred in mixed cultures

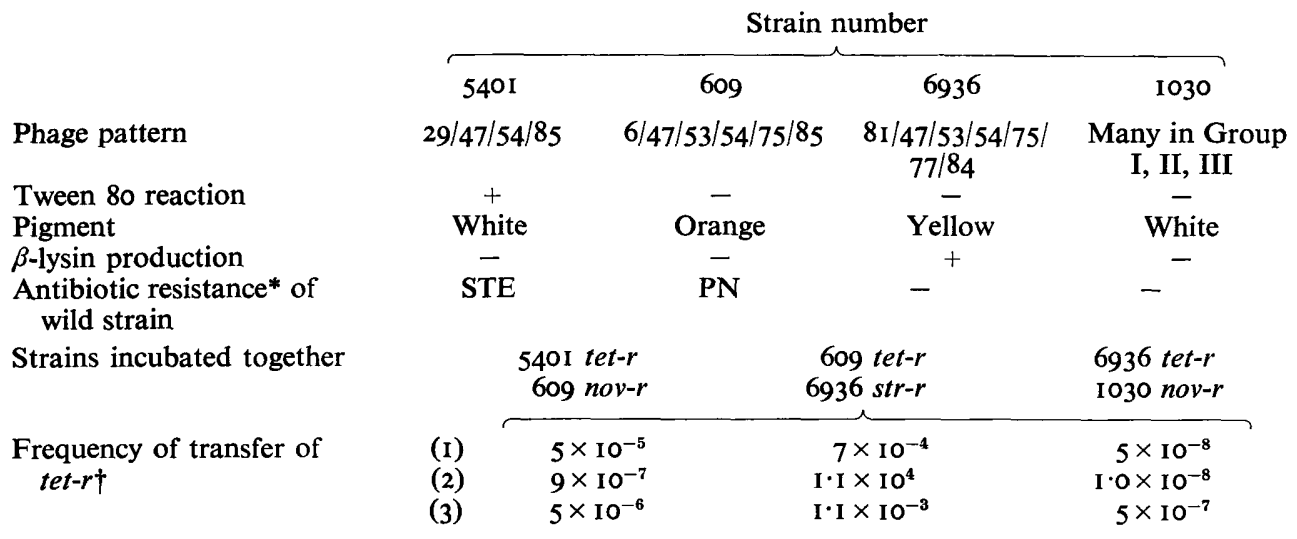
(N).

* Resistance to streptomycin (S), to tetracycline (T), to erythromycin (E), to penicillin (P), to neomycin $\dagger$ Experiments performed in triplicate.

whether P609 acts as a helper phage, the transducing particles being derived from another phage, or whether the particles are obtained from P609 itself. Evidence in favour of the latter was obtained by showing that P609 in a nonlysogenic strain (1030) also produced high-frequency transfer. Evidence that strain I030 wild-type and I030 tet-r are not detectably lysogenic is as follows. (i) Broth cultures of neither strain are inducible with mitomycin C or u.v. (by the method of Novick, 1967). The failure of mitomycin C to induce strains 1030 and I030 tet-r has been confirmed by Stickler (personal communication); (ii) strains I030 and 1030 tet-r have a very wide phage sensitivity and are lysed by all but 
two of the standard set of typing phages, although often at low efficiency. This is generally assumed to reflect the absence of prophages in the strain since these tend to reduce sensitivity to the typing phages; (iii) no phage activity against 30 test strains has been found in the filtrates of either culture.

No transfer of tetracycline-resistance from I030 tet-r to 6936 str-r occurred, but a P609 lysogen of 1030 tet-r transferred resistance to $6936 \mathrm{str}-r$ at high frequency $\left(2.5 \times 10^{-5}\right)$. Although it is possible to account for these results by the activation of cryptic defective phages in strains 1030 and 609 by P609, it seems more likely that the transducing particles are derived from P609 itself.

Additional evidence that the transferring particles were derived from P609 was obtained by showing that P6o9-resistant mutants of 6936 were no longer competent as recipients. The mutants were obtained by exposing the wild strain to nitrosoguanidine at $50 \mu \mathrm{g} . / \mathrm{ml}$. for 60 min. (Altenbern, 1967) and testing surviving colonies for resistance to P609. Six P609resistant colonies were obtained and none adsorbed phage P609 nor acquired tetracyclineresistance when incubated with 609 tet-r, but were lysed by some phages of the international typing set. Further mutagenesis of one P609-resistant 6936 colony gave about I \% of cocci susceptible to P609. These P609 sensitive revertants could also acquire resistance from 609 tet-r.

There was, therefore, a close correlation between the ability of 6936 to adsorb P609 and its capacity to act as a recipient. It seems extremely likely that the transducing particles were derived from P609 itself.

The high proportion of tetracycline-resistant recipients after $20 \mathrm{~h}$. incubation in mixed cultures is not due to a selective advantage acquired by rare resistant recipients. All of 80 tetracycline-resistant recipient colonies tested were still sensitive to P609. Moreover, the proportion of resistant and sensitive recipients in a mixed culture was shown to be constant over $20 \mathrm{~h}$.

The transfer of tetracycline-resistance at high frequency from 609 tet-r to 6936 str-r appears to be due to the release of transducing particles from 609 tet-r and their uptake by the recipient. Thus filtrates of 609 tet-r should contain many of these transducing particles.

Volumes of a 4-hour aerated culture of 609 tet-r in nutrient broth were filtered through a $0.45 \mathrm{~m} \mu$ millipore filter and added to the same volume of a 4-hour aerated culture of $6936 \mathrm{str}-r$ in calcium broth. After $30 \mathrm{~min}$. incubation at $37^{\circ}$, sodium citrate was added to a final concentration of $0.02 \mathrm{M}$ and the culture examined for transductants after a further $\mathrm{I} \frac{1}{2} \mathrm{~h}$. Filtrates of 609 tet-r contained about one transferring particle and about $10^{5}$ p.f.u. of P609 per $10^{10}$ cells (about $100 \mathrm{ml}$.). By contrast an unfiltered culture of 609 tet-r gave about $1^{2} 6936$ str-r tet-r/ml. A fivefold increase in the transfer rate was obtained by centrifuging the donor culture $(3000 \mathrm{~g}, 5 \mathrm{~min}$.) before filtration. These frequencies of transfer from filtrates seemed too low to account for the transfer of resistance in mixed cultures.

Attempts at transfer across asbestos, millipore or sintered glass filters were unsuccessful. But the absence of transfer could possibly be accounted for by calcium precipitates in the medium blocking the filter pores. The numbers of $\mathrm{P} 609$ phage particles passing filters during incubation on an orbital shaker were few. Another reason could be the need for highly aerobic conditions which were difficult to maintain in these experiments. The frequency fell tenfold if the mixed culture was incubated statically instead of with aeration. Similarly the volume of mixed culture in a $25 \mathrm{ml}$. screw-cap universal bottle greatly affected the frequency of transfer. The rate fell from about $\mathrm{I} \times \mathrm{IO}^{-4}$ with $5 \mathrm{ml}$. of culture to $4 \times 10^{-8} / \mathrm{ml}$. with $25 \mathrm{ml}$.

Thus although the transfer of tet-r may not have been demonstrable across filters because 
of difficulty in maintaining appropriate conditions, the failure to find transferring particles in the donor culture filtrates in sufficient numbers has not been explained. One of the following reasons seems likely, but the problem has not been resolved. The transducing phage particles are either largely cell-bound or very unstable; in either case cell-to-cell contact favours the transfer.

Phage P609 in various donor strains. Strain 609 tet-r was in most instances the donor strain associated with the highest rate of acquisition of resistance in the above recipient strains, probably because it harboured phage P609 which was absent from strains 6936 and 540I. The ability of phage P609 to act as a high-frequency transducing phage was investigated in five tetracycline-resistant recipient strains, the resistance having previously been transferred to each from strain 540I. The frequency of transfer of tetracycline-resistance to strain 6936 str-r from these cultures lysogenized with phage P609 was then compared with that from the cultures prior to lysogenization. The presence of phage P6og in each of the cultures increased the rate of acquisition of resistance in the recipient (Table 2).

Table 2. Effect of lysogenization with phage P609 of five donor strains on the transfer of tetracycline resistance to strain 6936 after $20 \mathrm{~h}$.

\begin{tabular}{|c|c|c|c|}
\hline \multirow[b]{2}{*}{ Donor strain } & \multirow[b]{2}{*}{$\begin{array}{c}\text { Lysogenic } \\
\text { state of } \\
\text { wild strain }\end{array}$} & \multicolumn{2}{|c|}{ Frequency of transfer } \\
\hline & & $\begin{array}{c}\text { Before } \\
\text { lysogenization } \\
\text { with P609 }\end{array}$ & $\begin{array}{c}\text { After } \\
\text { lysogenization } \\
\text { with } \mathrm{P} 609\end{array}$ \\
\hline 1030 tet-r & - & $<10^{-12}$ & $1 \cdot 0 \times 10^{-4}$ \\
\hline 6936 tet-r & + & $1 \cdot 0 \times 10^{-5}$ & $2 \times 10^{-4}$ \\
\hline PS 83A tet-r & + & $<\mathrm{IO}^{-12}$ & $8 \times 10^{-6}$ \\
\hline PS 47 tet-r & + & $8 \times 10^{-7}$ & $5 \times 10^{-5}$ \\
\hline PS 54 tet-r & + & $5 \times 10^{-7}$ & $1 \cdot 5 \times 10^{-5}$ \\
\hline
\end{tabular}

Table 3. Effect of growth at $37^{\circ}, 42^{\circ}$ and storage at room temperature for 3 months on the loss of resistance to tetracycline in strains $540 \mathrm{I}$ tet-r, 609 tet-r and 5936 tet-r

\begin{tabular}{|c|c|c|c|c|c|c|}
\hline \multirow[b]{2}{*}{$\begin{array}{l}\text { Conditions of } \\
\text { of culture }\end{array}$} & \multicolumn{2}{|c|}{5401 tet-r } & \multicolumn{2}{|c|}{609 tet $-r$} & \multicolumn{2}{|c|}{6936 tet-r } \\
\hline & $\begin{array}{c}\text { No. } \\
\text { colonies } \\
\text { screened }\end{array}$ & $\begin{array}{l}\text { No. } \\
\text { sensitive } \\
\text { to tetra- } \\
\text { cycline }\end{array}$ & $\begin{array}{c}\text { No. } \\
\text { colonies } \\
\text { screened }\end{array}$ & $\begin{array}{l}\text { No. } \\
\text { sensitive } \\
\text { to tetra- } \\
\text { cycline }\end{array}$ & $\begin{array}{c}\text { No. } \\
\text { colonies } \\
\text { screened }\end{array}$ & $\begin{array}{c}\text { No. } \\
\text { sensitive } \\
\text { to tetra- } \\
\text { cycline }\end{array}$ \\
\hline $37^{\circ}$ (overnight) & 2644 & 0 & 2783 & 0 & 2725 & o \\
\hline $42^{\circ}$ (overnight) & 2168 & 2 & 2245 & I & 2901 & 2 \\
\hline Room temp. 3 months & 2692 & 3 & 2969 & I & 2452 & I \\
\hline
\end{tabular}

Evidence of a plasmid location for the tetracycline-resistance determinant. To establish the location of the genes for tetracycline-resistance in 540I tet-r, 609 tet-r and 6936 tet-r, I looked for segregation of tet-r clones from these strains and studied the effect of u.v. light on the frequency of transduction from lysates propared from each isolate.

Cultures were plated for single colonies and replica-plated to agar plates containing Io $\mu \mathrm{g}$. tetracycline $/ \mathrm{ml}$. In each culture examined, tetracycline-resistance was lost at a high frequency $\left(\mathrm{IO}^{-3}\right)$ after storage for 3 months at room temperature, or overnight growth at $42^{\circ}$, but not at $37^{\circ}$ (Table 3). The frequent appearance of tet-s segregants indicates that the 
gene(s) for tetracycline resistance is carried on a plasmid in all three strains. Further evidence for this conclusion was obtained by studying the effect of u.v. on the transduction frequencies of mitomycin C-induced lysates. Strain 6936 was the recipient strain for lysates from 5401 tet-r and strain 609 for those from 609 tet-r and 6936 tet-r. There was an exponential loss in both plaque-forming titre and transduction frequency in the lysate from all three strains with increasing u.v. dose (Fig. 2). These curves are typical for plasmid-borne markers (e.g. Arber, 1960; Asheshov, 1966, 1969; Poston, 1966).

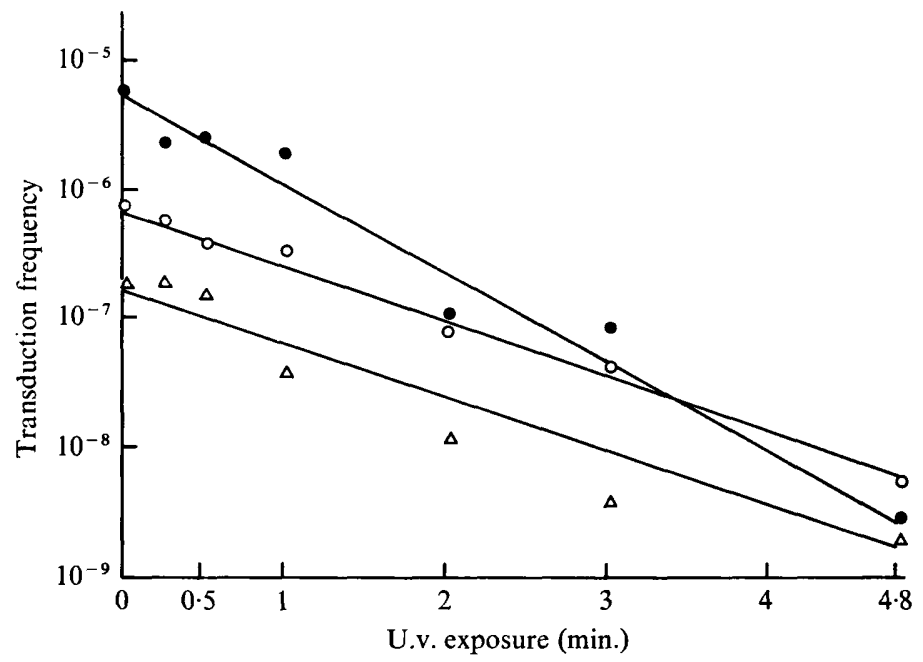

Fig. 2. Effect of u.v. exposure on the transduction frequency of tetracycline-resistance from mitomycin $\mathrm{C}$-induced lysates from three cultures. The transduction frequency is expressed as the number of transductants per unirradiated phage titre. - , Culture 609 tet-r as donor; $O-O$, culture 6939 tet $-r ; \triangle-\triangle$, culture 540I tet-r. Strain 609 was the recipient for donor cultures 609 tet-r and 6936 tet-r and strain 6936 recipient for $540 \mathrm{I}$ tet-r.

Table 4. Proportion of recipient strains acquiring tetracycline-resistance after incubation for $20 \mathrm{~h}$. with each of three donor strains

Recipient strains (and phage pattern)

$$
\begin{aligned}
& (29 / 77) \\
& (52 \mathrm{~A} / 77) \\
& (6 / 47 / 54 / 75) \\
& (\mathrm{N} . \mathrm{T} .) \\
& \text { (77) } \\
& \text { Strain 540I tet-s } \\
& \quad \text { (see Table I) }
\end{aligned}
$$

Strain 609 (see Table 1) Strain 6936 (see Table I)
Donor strains

\begin{tabular}{ccc}
\hline 5401 tet-r & 609 tet-r & 6936 tet-r \\
$7 \times 10^{-8}$ & $5 \times 10^{-6}$ & $<10^{-10}$ \\
$<10^{-10}$ & $9 \times 10^{-7}$ & $1 \times 10^{-8}$ \\
$9 \times 10^{-6}$ & $8 \times 10^{-5}$ & $2 \times 10^{-6}$ \\
$<10^{-10}$ & $3 \times 10^{-8}$ & $9 \times 10^{-9}$ \\
$<10^{-10}$ & $6 \times 10^{-7}$ & $<10^{-10}$ \\
$9 \times 10^{-8}$ & $4 \times 10^{-7}$ & $5 \times 10^{-8}$ \\
& & \\
$5 \times 10^{-6}$ & $1 \cdot 2 \times 10^{-6}$ & $3 \times 10^{-7}$ \\
$7 \times 10^{-5}$ & $7 \times 10^{-4}$ & $1 \times 10^{-5}$
\end{tabular}

N.T. = Non typable.

\section{Transfer of tetracycline-resistance to other recipient strains}

If a significant number of naturally occurring tetracycline-resistant strains of Staphylococcus aureus had aquired the resistance by a transfer mechanism, this gene(s) should be transferable in vitro to a wide variety of strains. Strains 540I tet-r, 609 tet-r and 6936 tet-r were each incubated with eight different recipient strains. Tetracycline-resistance was 
transferable from 609 tet- $r$ to all eight recipients, from 6936 tet-r to six and from 540 I tet-r to five (Table 4). The failure of 540I tet-r and 6936 tet-r to transfer tetracycline-resistance to all of the recipients might be accounted for by insusceptibility of these recipients to the particular transducing phage.

\section{DISCUSSION}

Mutants showing the same level of resistance to tetracycline as clinical strains rarely, if ever, arise de novo from sensitive strains in vitro (R. W. Lacey, unpublished observations). Yet resistance to tetracycline is widespread in staphylococci (e.g. Mitsuhashi, Oshima, Kawaharada \& Hashimoto, 1965). Mechanisms additional to mutation and selection may therefore account for the spread of resistance, particularly since wild strains are almost always sensitive to the drug (Birnstingl, Shooter \& Hunt, 1952; Elek, 1959).

Of these other mechanisms, transfer of tetracycline-resistance by transduction in Staphylococcus aureus has been studied in vitro (Pattee \& Baldwin, 196I ; Mitsuhashi et al. 1965; McDonald, 1966; Poston, 1966) and in mice after injection of the recipient strain followed by the phage (Jarolmen, Bondi \& Crowell, 1965). In those studies a cell-free phage preparation induced from a tetracycline-resistant strain was employed as the source of transducing particles.

The frequency of transfer of antibiotic-resistance by these phage preparations is low often between $I$ in $10^{6}$ and $10^{9}$ of the particles confer resistance to an appropriate recipient strain. Since cultures of lysogenic staphylococci usually contain less than $10^{5}$ phage particles/ml. (Rountree, 1949), it is not surprising that filtrates, as were found here, are poor in transducing ability. This has led some authors (e.g. Richmond, 1969) to question the transfer of resistance by this type of transduction under natural conditions.

This paper demonstrates that transfer of tetracycline-resistance occurs in mixed cultures and is almost certainly by transduction. The transfer occurs at times at high frequency, despite the few transducing elements in the donor culture filtrate. Instability or cell-binding of the transducing particles are possible explanations for the failure to detect them in culture filtrates.

Strain 6936 was the recipient strain that acquired tetracycline-resistance at the highest frequency from several donor organisms and was the same strain to which neomycin-resistance was transferred at greatest frequency (Lacey, I97I). Strain 6936 possesses many features of staphylococci recently epidemic in hospitals (Jevons, John \& Parker, I966). Both strain 6936 and many of these hospital isolates produce yellow pigment and $\beta$ lysin, but not the Tween 80 lipase, although strain 6936 is sensitive to all the commonly used antistaphylococcal antibiotics. The facility with which a strain can take up resistant determinants could affect its survival in the hospital environment where antibiotics are widely used; and it is possible that many staphylococci owe their survival to their ability to accept transducing particles.

I am grateful to Professor H. M. Richmond for helpful advice and criticism.

\section{REFERENCES}

Altenbern, R. A. (1967). Genetic studies of pigmentation of Staphylococcus aureus. Canadian Journal of Microbiology I3, 389-395.

ARBer, W. (1960). Transduction of chromosomal genes and episomes in Escherichia coli. Virology II, 273288.

Asheshov, E. H. (1966). Chromosomal location of the genetic elements controlling penicillinase production in a strain of Staphylococcus aureus. Nature, London 210, 804-806. 
AsHeshov, E. H. (1969). The genetics of penicillinase production in Staphylococcus aureus strain PS 80. Journal of General Microbiology 59, 289-30I.

BiRnstingl, M. A., Shooter, R. A. \& HUNT, M. F. (1952). Sensitivity to five antibiotics of strains of Staph. pyogenes isolated from out-patients. British Medical Journal 2, 253-254.

Blair, J. E. \& Williams, R. E. O. (1961). Phage typing of staphylococci. Bulletin of the World Health Organisation 24, 771-784.

BüLow, P. (1970). A new epidemic phage type of Staphylococcus aureus. 5. Epidemic spread of phages among Danish hospital staphylococci. Acta pathologica et microbiologica scandinavica 78B, 29-40.

DyKe, K. G. H., PARKeR, M. T. \& Richmond, H. M. (1970). Penicillinase production and metal-ion resistance in Staphylococcus aureus cultures isolated from hospital patients. Journal of Medical Microbiology 3, $125-136$.

ELEK, S. D. (1959). Staphylococcus pyogenes and its relation to Disease, p. 472. Edinburgh: Livingstone.

GoNZALEZ, C. \& SIERRA, G. (196I). Lipolyte activity of some anaerobic bacteria. Nature, London 189, 601602.

Jarolmen, H., Bond, A. \& Crowell, R. L. (1965). Transduction of Staphylococcus aureus to tetracycline resistance in vivo. Journal of Bacteriology 89, $1286-1290$.

Jevons, M. P., John, M. \& Parker, M. T. (I966). Cultural characters of a newly recognized group of hospital staphylococci. Journal of Clinical Pathology 19, 305-312.

LACEY, R. W. (197I). High-frequency transfer of neomycin resistance between naturally-occurring strains of Staphylococcus aureus. Journal of Medical Microbiology 4, 73-84.

LACEY, R. W., ALder, V. G. \& GILleSPIE, W. A. (1970). The survival of Staphylococcus aureus on human skin. An investigation using mixed cultures. British Journal of Experimental Pathology 51, 305-313.

MCDonALD, S. (1966). Transduction of antibiotic resistance in Staphylococcus aureus. Lancet 2, I 107-I 110.

MitsuHaShi, S., Oshima, H., KaWAHARAdA, U. \& HaShimoto, H. (1965). Drug resistance of staphylococci: 1. Transduction of tetracycline resistance with phage-lysates obtained from multiply resistant staphylococci. Journal of Bacteriology 89, 967-976.

Novick, R. P. (1967). Properties of a cryptic high-frequency transducing phage in Staphyloccocus aureus. Virology 33, $155-166$.

Novick, R. P. \& MoRSE, S. I. (1967). In vivo transmission of drug resistance factors between strains of Staphylococcus aureus. Journal of Experimental Medicine 125, 45-59.

Pattee, P. A. \& Baldwin, J. N. (I96I). Transduction of resistance to chlortetracycline and novobiocin in Staphylococcus aureus. Journal of Bacteriology 82, 875-881.

Poston, S. M. (1966). Cellular location of the genes controlling penicillinase production and resistance to streptomycin and tetracycline in a strain of Staphylococcus aureus. Nature, London 210, 802-804.

REPORT of the sub-committee on phage-typing of staphylococci of the international committee on nomenclature of bacteria, Moscow, July 1966 (1967). International Journal of Systemic Bacteriology 17 , 1 1 3-1 25.

RichmoND, M. H. (1969). Extrachromosomal elements and the spread of antibiotic resistance in bacteria. Biochemical Journal 1r3, 225-234.

RountreE, P. M. (1949). The phenomenon of lysogenicity in staphylococci. Journal of General Microbiology 3, $153-163$.

ROUNTREE, P. M. (195I). The role of certain electrolytes in the adsorption of staphylococcal bacteriophages. Journal of General Microbiology 5, 673-680.

Williams, R. E. O. \& Rippon, J. E. (1952). Bacteriophage typing of Staphylococcus aureus. Journal of Hygiene 50, 320-353.

WirLIS, A. T. \& TURNER, G. C. (1963). Staphyloccoci in the hospital environment. Journal of Pathology and Bacteriology 85, 395-405. 